\title{
Efeitos combinados da restrição alimentar e flushing sobre a fertilidade de marrãs inseminadas artificialmente em diferentes ciclos estrais
}

\author{
Combined effects feeding restriction and flushing on fertility of gilts \\ inseminated at different estrous cycles
}

\author{
Adriana MUNIZ ${ }^{1}$; Gisele Mouro RAVAGNANI ${ }^{1}$; Simone Maria Massami Kitamura MARTINS ${ }^{1}$; \\ André Furugen Cesar de ANDRADE ${ }^{1,2}$; Aníbal de Sant'Anna MORETTI ${ }^{1}$ \\ ${ }^{1}$ Laboratório de Pesquisa em Suínos (LPS) do Departamento de Nutrição e Produção Animal da \\ Faculdade de Medicina Veterinária e Zootecnia da Universidade de São Paulo, São Paulo - SP, Brasil \\ ${ }^{2}$ Departamento de Reprodução Animal da Faculdade de Medicina Veterinária e Zootecnia \\ da Universidade de São Paulo, São Paulo - SP, Brasil
}

\begin{abstract}
Resumo
Foi avaliado o efeito da restrição alimentar na fase pré-púbere associada ao emprego do "flushing” no ciclo precedente ao da inseminação artificial, sendo realizada no $2^{\circ}$ ou $3^{\circ}$ estro, sobre o desempenho produtivo, número de corpos lúteos e de fetos, além da taxa de sobrevivência fetal. Um total de 96 fêmeas foi distribuído em delineamento inteiramente casualizado com arranjo fatorial $2 \times 2 \times 2$, sendo considerados os fatores: estratégia nutricional, alimentação ad libitum ou restrita; utilização ou não de "flushing" no ciclo precedente a IA, e momento da IA, realizada no $2^{\circ}$ ou $3^{\circ}$ estro, constituindo oito tratamentos. A idade em que as marrãs manifestaram o $1^{\circ}$ estro não foi influenciada pela estratégia nutricional, mas o peso e o ganho de peso foram maiores $(\mathrm{P}<0,05)$ na alimentação ad libitum. Não foram observados interação e tão pouco efeito de tratamento no número de corpos lúteos e a taxa de sobrevivência dos fetos. O número de fetos em fêmeas mantidas em alimentação ad libitum não foi influenciado pelo momento da IA, contudo as fêmeas em restrição alimentar apresentaram aumento $(\mathrm{P}<0,05)$ no número de fetos quando inseminadas no $3^{\circ}$ estro. Desse modo, conclui-se que o emprego da estratégia nutricional ad libitum possibilita adiantar a inseminação para o $2^{\circ}$ estro sem redução no número de fetos, diminuindo os dias não produtivos da marrã. Porém, essa estratégia reduz a taxa de sobrevivência fetal até $35^{\circ}$ dia de gestação.
\end{abstract}

Palavras-chave: Marrãs. Flushing. Restrição alimentar.

\begin{abstract}
The study evaluated the effect of dietary restriction in prepubertal gilts associated with the use of flushing in the preceding cycle of artificial insemination, $2^{\text {nd }}$ or $3^{\text {rd }}$ estrus on productive performance, number of corpora lutea and fetus, besides the fetus survival rate. A total of 96 gilts were distributed to a completely randomized design with $2 \times 2 \times 2$ factorial arrangement of treatments, consisting of: nutritional strategy, ad libitum or restricted feeding, use or not of flushing in the preceding cycle AI, and time of AI, held on the $2^{\text {nd }}$ or $3^{\text {rd }}$ estrus. The age at which gilts expressed the 1 st estrus was not influenced by nutritional strategy, but the body weight and weight gain were higher $(\mathrm{P}<0.05)$ in gilts submitted to ad libitum feeding. No interactions were observed as treatment effect on number of corpora lutea and fetus survival rate. The number of fetuses in females on ad libitum feeding was not influenced by time of AI, but the females feed restriction showed an increase $(\mathrm{P}<0.05)$ in the number of fetuses when inseminated on $3^{\text {rd }}$ estrus. Thus, it is concluded that the use of ad libitum nutritional strategy allows for insemination forward $2^{\text {nd }}$ estrus without reducing the number of fetuses, reducing the non-productive days gilt. However, this strategy reduces the rate of fetal survival until day 35 of gestation.
\end{abstract}

Keywords: Gilts. Flushing. Food restriction.

\section{Introdução}

O manejo das marrãs nas fases pré e pós-púbere tem despertado o interesse de pesquisadores e produtores, uma vez que as linhagens genéticas atuais são mais prolíficas e apresentam maior deposição de tecido magro na carcaça, contudo a capacidade de consumo

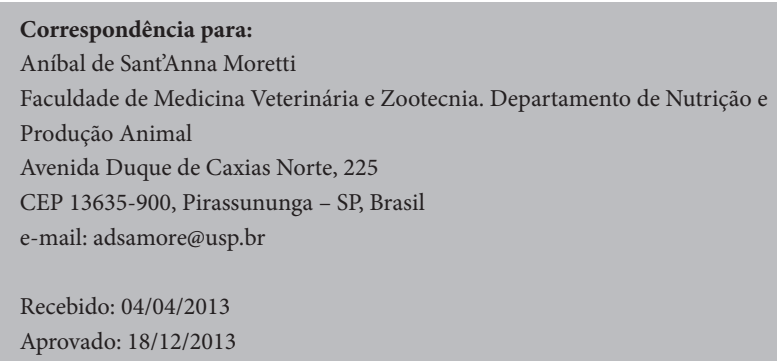


de ração ainda é limitada (THAKER; BILKEI, 2005). Esta última condição é importante durante o primeiro aleitamento, situação na qual há mobilização de reservas corporais para atender a demanda nutricional para a produção de leite (KIM; EASTER, 2001).

Quando a fêmea desenvolve o processo de catabolismo intenso durante o primeiro aleitamento, ocorre impacto negativo sobre a vida útil reprodutiva, o que pode ser caracterizado pelo aumento do intervalo desmame-estro (TANTASUPARUK et al., 2001), por reduções nas taxas de ovulação, na sobrevivência embrionária (KEMP; SOEDE, 2004) e no tamanho da leitegada subsequente (TANTASUPARUK et al., 2001).

Tais motivos justificam o manejo específico com a marrã, pois, após a manifestação da puberdade, esta deve apresentar de dois a quatro estros antes da cobertura, para que ocorra o desenvolvimento dos órgãos genitais (tamanho e peso do útero) (VIANNA et al., 2004), além de ser relacionado ao aumento do tamanho da primeira leitegada (FOXCROFT; AHERNE, 2000) . A precocidade da puberdade é influenciada por diversos fatores, entre eles o genético, a idade, o peso corporal, o estado metabólico, a temperatura ambiente e a exposição ao macho (HUGHES, 1982).

A utilização de um manejo nutricional inadequado em uma das fases do ciclo reprodutivo da fêmea pode influenciar vários aspectos da biologia reprodutiva (FOXCROFT, 1997). O emprego durante sete dias de uma alimentação inadequada (restrita), de modo a atender as exigências de mantença, inibiu quase que totalmente a secreção do hormônio luteinizante (LH), mas não teve maior impacto na secreção do hormônio folículo estimulante (FSH) (ALMEIDA et al., 2012). $\mathrm{O}$ posterior fornecimento da alimentação à vontade resultou na imediata restauração da secreção de $\mathrm{LH}$, e após sete dias de alimentação à vontade foi observado um aumento no desenvolvimento folicular ovariano.

Uma estratégia conhecida para melhorar a reprodução das marrãs é a utilização do "flushing" alimentar (COSGROVE et al., 1995). Rhodes, Davis e Stevenson (1991) o definiram como o aumento de consumo de alimento, durante o período pré-cobertura, levando a mudanças metabólicas. Essas mudanças parecem estar ligadas à energia ingerida pela dieta e, especialmente em marrãs, parece haver uma relação positiva entre o nível de energia ingerida e a taxa de ovulação (FAILLACE; BIGGS; HUNTER, 1994).

A utilização do "flushing" alimentar está associada às alterações plasmáticas de concentrações séricas hormonais, que levam ao aumento no número de ovulações (BELTRANENA et al., 1991b) e melhora da viabilidade embrionária (LAGO et al., 2004). O "flushing" empregado no primeiro ciclo estral em marrãs influenciou positivamente a viabilidade embrionária (BELTRANENA et al., 1991a; YOUNG, 1998).

Diante do exposto, o presente trabalho avaliou o efeito da restrição alimentar na fase pré-púbere associada ao emprego do "flushing" alimentar no ciclo precedente ao da inseminação artificial, realizada no $2^{\circ}$ ou $3^{\circ}$ estro, sobre o desempenho produtivo, número de corpos lúteos e de fetos, além da taxa de sobrevivência fetal.

\section{Materiais e Métodos \\ Local e animais}

O experimento foi conduzido nas instalações experimentais do Laboratório de Pesquisa em Suínos (LPS) da FMVZ / USP. Foram utilizadas 96 marrãs pré-púberes de linhagem híbrida comercial (C22, Agroceres ${ }^{\text {PIC }}$, Brasil), com idade de 94,5 \pm 4,39 dias e peso médio de 46,36 $\pm 5,40 \mathrm{~kg}$. As fêmeas foram alojadas em baias coletivas com 1,5 $\mathrm{m}^{2} /$ animal, comedouro semiautomático, bebedouro tipo chupeta e presença de lâmina d’água.

\section{Delineamento e distribuição nos tratamentos}

O delineamento experimental foi inteiramente casualizado com arranjo fatorial de tratamentos $2 \times 2 \times 2$, sendo considerados os fatores: estratégia nutricional, alimentação ad libitum (A) ou restrita (R); utilização 
de "flushing" no ciclo precedente a cobertura, com (cF) ou sem (sF) e momento da inseminação artificial, realizada no $2^{\circ}(2)$ ou $3^{\circ}(3)$ estro, constituindo, assim oito tratamentos: (AcF2) alimentação ad libitum com "flushing" e IA no $2^{\circ}$ estro, (AcF3) alimentação ad libitum com "flushing" e IA no $3^{\circ}$ estro, (AsF2) alimentação ad libitum sem "flushing" e IA no $2^{\circ}$ estro, (AsF3) alimentação ad libitum sem "flushing" e IA no $3^{\circ}$ estro, (RcF2) alimentação restrita com "flushing" e IA no $2^{\circ}$ estro, $(\mathrm{RcF} 3)$ alimentação restrita com "flushing" e IA no $3^{\circ}$ estro, (RsF2) alimentação restrita sem "flushing" e IA no $2^{\circ}$ estro, (RsF3) alimentação restrita sem "flushing" e IA no $3^{\circ}$ estro, totalizando 12 repetições por tratamento.

A alimentação restrita foi fornecida com redução em $20 \%$ a partir da quantia correspondente ao consumo ad libitum. As fêmeas foram alimentadas com ração de recria $(3.241,49 \mathrm{kcal} / \mathrm{kg}$ EM e 18,68\% PB) do início do estudo aos 120 dias e dos 120 dias até a inseminação artificial as fêmeas foram alimentadas com ração de reposição (3.115,23 kcal/kg EM e 17,37\% PB).

Os diagnósticos do $1^{\circ}$ estro, assim como dos subsequentes $\left(2^{\circ}\right.$ e $\left.3^{\circ}\right)$, foram realizados por meio da introdução do macho nas baias das fêmeas, conduzido duas vezes ao dia por quinze minutos. Essa introdução do macho nas baias foi iniciada por volta dos 155 dias de idade das marrãs. O "flushing" alimentar foi empregado nos últimos 14 dias antes da inseminação artificial (IA) (48 fêmeas no $2^{\circ}$ estro e 48 fêmeas no $3^{\circ}$ estro), utilizando-se uma ração com $3.138,08 \mathrm{kcal} /$ kg EM e 17,45\% PB, e com farelo de trigo, fornecida ad libitum. Os animais sem "flushing" continuaram a receber a ração de reposição $(3.115,23 \mathrm{kcal} / \mathrm{kg}$ EM e $17,37 \% \mathrm{~PB})$, respeitando a estratégia nutricional. Todas as fêmeas foram inseminadas no momento em que foi verificado que as mesmas estavam em estro, sendo as inseminações posteriores em intervalos de $12 \mathrm{~h}$ até o final do estro. Após a inseminação, os animais passaram a receber a ração de gestação $(2 \mathrm{~kg} /$ animal, 2.834,01 kcal/kg EM e 17,45\% PB) até os 35 dias, período no qual as fêmeas foram abatidas.

\section{Variáveis avaliadas}

O desempenho das fêmeas foi avaliado por meio de peso individual, idade e ganho médio diário de peso desde os 94 dias até a manifestação do $1^{\circ}$ estro. Aos 35 dias de gestação, as fêmeas foram abatidas para a verificação do número de corpos lúteos e de fetos. Anteriormente à eletronarcose, foi introduzida na cérvix das fêmeas uma pipeta de inseminação artificial preenchida com silicone, destinada a evitar o refluxo de urina para o interior do útero e assim prevenir possíveis danos à integridade dos fetos. $\mathrm{O}$ processo de retirada dos órgãos genitais foi efetuado logo após a sangria, através de uma incisão de aproximadamente dez centímetros sobre a linha branca na região inguinal. Em seguida, os cornos uterinos e os ovários foram dissecados para averiguação da presença de corpos lúteos, contagem do número de fetos e cálculo da taxa de sobrevivência fetal (número de fetos/número de corpos lúteos x 100).

As variáveis desempenho, número de corpos lúteos, número de fetos e taxa de sobrevivência fetal foram analisadas com o programa computacional Statistical Analysis System (SAS, 1998).

O modelo experimental foi o seguinte: $Y=\mu+A_{i}$ $+\mathrm{F}_{\mathrm{j}}+\mathrm{E}_{1}+(\mathrm{AFE})_{\mathrm{ijl}}+\mathrm{e}_{\mathrm{ij} \text {. }}$ No qual, Y é a observação do tratamento $\mathrm{i}, \mathrm{f}$ e $\mathrm{l}, \mu$ é a média geral, $\mathrm{A}_{\mathrm{i}}$ é o efeito do tratamento i, $\mathrm{F}_{\mathrm{j}}$ é o efeito do tratamento $\mathrm{j}, \mathrm{E}_{1}$ é o efeito do tratamento $1,(\mathrm{AFE})_{\mathrm{ij} l}$ é o efeito de interação entre os tratamentos $\mathrm{i}$, j e l e e $\mathrm{i}_{\mathrm{ij} 1}$ é o erro.

Foram verificadas a normalidade dos resíduos e a homogeneidade das variâncias para as variáveis analisadas. Os dados que atenderam a essas premissas foram submetidos à análise de variância pelo procedimento GLM (PROC GLM). O nível de significância foi de $5 \%$.

\section{Resultados}

\section{Desempenho à puberdade}

A idade em que as marrãs manifestaram o $1^{\circ}$ estro não foi influenciada $(\mathrm{P}>0,05)$ pela estratégia nutri- 
cional, alimentação ad libitum ou restrita (Tabela 1). Já o peso corporal e o ganho médio diário de peso foram maiores $(\mathrm{P}<0,05)$ para animais com alimentação ad libitum (Tabela 1). Das 96 fêmeas que iniciaram o estudo, 94,79\% manifestaram o $1^{\circ}$ estro após estimulação realizada com o macho.

\section{Número de corpos lúteos}

Não foi verificada interação entre os tratamentos nesta característica analisada $(\mathrm{P}>0,05)$. As fêmeas submetidas à restrição alimentar apresentaram 16,74 $\pm 2,70$ corpos lúteos em comparação com as fêmeas alimentadas ad libitum com $15,81 \pm 2,87$, sendo constatado aumento $(\mathrm{P}<0,05)$ de 0,93 corpos lúteos nas fêmeas mantidas em restrição. O "flushing" alimentar não exerceu efeito sobre o número de corpos lúteos. Em relação ao momento da IA, foi observado que as fêmeas inseminadas no $3^{\circ}$ estro apresentaram maior $(\mathrm{P}<0,05)$ número de corpos lúteos $(16,71 \pm 2,77)$ em comparação às inseminações realizadas no $2^{\circ}$ estro $(15,84 \pm 2,82)$.

\section{Número de fetos}

Não foi observada interação entre os tratamentos sobre o número de fetos $(\mathrm{P}>0,05)$. Verificou-se interação $(\mathrm{P}<0,05)$ entre momento da IA e a estratégia nutricional, sendo que o mesmo não foi observado entre os demais tratamentos. Nas fêmeas mantidas em alimentação ad libitum, o número de fetos não foi influenciado pelo momento da IA, contudo as fêmeas em restrição alimentar apresentaram aumento $(\mathrm{P}<0,05)$ no número de fetos, quando inseminadas no $3^{\circ}$ estro $\left(10,30 \pm 2,43\right.$ vs. $12,96 \pm 2,63,2^{\circ}$ e $3^{\circ}$ estro, respectivamente) (Tabela 2 ).

\section{Taxa de sobrevivência fetal}

Nesta característica, não foi verificada interação $(P>0,05)$ entre os tratamentos. Em relação ao efeito da estratégia nutricional empregada, a restrição alimentar aumentou $(\mathrm{P}<0,05)$ o percentual de sobrevivência fetal, $(78,21 \% \pm 18,22)$ em comparação com a alimentação ad libitum $(74,94 \% \pm 21,35)$. Adicionalmente, as fêmeas inseminadas no $3^{\circ}$ estro apresen-

Tabela 1 - Desempenho produtivo de marrãs alimentadas ad libitum e sob restrição quanto a manifestação do $1^{\circ}$ estro $^{1}$ - Pirassununga - SP - 2013

$\begin{array}{lcc}\text { Características } & \begin{array}{c}\text { Ad libitum } \\ \mathbf{n}=\mathbf{4 8}\end{array} & \text { Restrição } \\ \mathrm{n}=48 & & 165,91 \pm 11,75 \\ \text { Idade }(\mathrm{d}) & 163,35 \pm 7,02 & 105,25 \pm 14,70^{\mathrm{b}} \\ \text { Peso }(\mathrm{kg}) & 113,89 \pm 15,1^{\mathrm{a}} & 0,84 \pm 0,12^{\mathrm{b}} \\ \text { GMDP }(\mathrm{g} / \mathrm{d}) & 0,94 \pm 0,12^{\mathrm{a}} & \\ & & \\ { }^{1} \text { Tratamentos apresentados média } \pm \text { desvio-padrão } & \\ \text { a-b Valores com letras diferentes sobrescritas dentro da mesma linha são diferentes }(\mathrm{P}<0,05) \text { pelo Anova } \\ \text { GMDP - ganho médio diário de peso }\end{array}$

Tabela 2 - Número de fetos analisados aos 35 dias de gestação de marrãs alimentadas ad libitum e sob restrição, inseminadas no $2^{\circ}$ ou $3^{\circ}$ estro ${ }^{1}$ - Pirassununga SP - 2013

$\begin{array}{lcc}\text { Momento da IA } & \text { Ad libitum } & \text { Restrição } \\ & 12,77 \pm 2,24^{\mathrm{a}, \mathrm{A}} & 10,30 \pm 2,43^{\mathrm{b}, \mathrm{A}} \\ 2^{\circ} \text { estro } & 12,89 \pm 2,85^{\mathrm{a}, \mathrm{A}} & 12,96 \pm 2,63^{\mathrm{a}, \mathrm{B}} \\ 3^{\circ} \text { estro } & \\ { }^{1} \text { Tratamentos apresentados média } \pm \text { desvio-padrão } & \\ { }^{\mathrm{a}, \mathrm{b}} \text { Valores com letras minúsculas diferentes sobrescritas dentro da mesma linha são diferentes }(\mathrm{P}<0,05) \\ \text { pelo Anova } \\ \text { A,B Valores com letras maiúsculas diferentes sobrescritas dentro da mesma coluna são diferentes }(\mathrm{P}<0,05) \\ \text { pelo Anova }\end{array}$


taram maiores $(\mathrm{P}<0,05)$ taxas de sobrevivência fetal $(78,14 \% \pm 16,48)$ comparadas às inseminadas no $2^{\circ}$ estro $(75,00 \% \pm 22,65)$.

\section{Discussão}

No presente trabalho, as fêmeas submetidas à restrição alimentar manifestaram o $1^{\circ}$ estro aos 165,91 dias de idade, pesando 105,25 kg. Newton e Mahan (1992) avaliaram a utilização de três percentuais de restrição alimentar (50\%, 25\% e $0 \%$ do ad libitum) e observaram que as fêmeas tratadas com restrição alimentar de $25 \%$ do ad libitum manifestaram estro aos 223,8 dias de idade com peso de 123,6 kg. O resultado obtido por Newton e Mahan (1992) corrobora com os descritos por Le Cozler et al. (1999), que ao realizarem restrição alimentar de 0,8 da ad libitum, as fêmeas também manifestaram $1^{\circ}$ estro tardiamente, aos 203,2 dias de idade, com peso superior, $117 \mathrm{~kg}$, ao obtido no presente estudo.

Embora as fêmeas do presente trabalho estivessem com peso inferior aos relatados por Newton e Mahan (1992) e Le Cozler et al. (1999), as mesmas manifestaram o $1^{\circ}$ estro com 57,89 dias e 37,29 dias de antecedência, respectivamente, apresentando uma precocidade equivalente a 2,76 e 1,77 ciclos estrais. A precocidade observada no presente trabalho pode ter sido influenciada por fatores como a genética, a idade, o peso corporal, o estado metabólico e a exposição ao macho (HUGHES, 1982). Van Wettere et al. (2006) referem que a partir de 160 dias de idade as fêmeas expostas ao cachaço sexualmente maduro, apresentaram maior sincronização da puberdade.

A marrã deve atingir precocemente a puberdade, pois isto propicia a manifestação de um maior número de ciclos estrais antes da cobertura, desenvolvimento dos órgãos genitais, em tamanho e peso do útero (VIANNA et al., 2004) e aumento do tamanho da primeira leitegada (FOXCROFT; AHERNE, 2000). Os resultados do presente trabalho são similares aos obtidos por Foxcroft e Aherne (2000) e Vianna et al. (2004), uma vez que houve maior número de corpos lúteos e fetos nas fêmeas inseminadas no terceiro estro em comparação com aquelas inseminadas no segundo.

Ao se considerar apenas a estratégia nutricional restritiva, Beltranena et al. (1991a) observaram menor número de corpos lúteos (12) que o observado neste estudo (16). Essa diferença pode estar relacionada a distintos fatores (BURNETT; WALKER, 1988; CHRISTENSON, 1993; BOOTH; COSGROVE; FOXCROFT, 1996; CHAMBERLAIN; HUGHES, 1996) como a linhagem, o estro considerado, além do regime alimentar. $\mathrm{O}$ maior número de fetos foi obtido em marrãs tratadas com estratégia nutricional restritiva associada a IA no $3^{\circ}$ estro. Já a quantidade de fetos das fêmeas alimentadas ad libitum parece não ter sido influenciada pelo momento da IA.

Quando analisada apenas a estratégia nutricional, constata-se o aumento de 1,24 fetos em animais submetidos à alimentação com restrição em comparação ao grupo ad libitum. Diversas investigações indicam haver interação positiva entre a restrição alimentar e o "flushing" (EDWARDS et al., 1968; BELTRANENA et al., 1991b; YOUNG, 1998), sendo observado que o emprego do "flushing" alimentar, após sete a oito dias de restrição, melhorou a qualidade do oócito e a taxa de sobrevivência dos fetos. No presente trabalho, essa mesma interação positiva não foi observada, provavelmente devido à restrição ter sido empregada desde a fase pré-púbere até os primeiros sete dias do estro de cobertura, seguida pela substituição da alimentação fornecida ad libitum por 14 dias subsequentes, sendo que, nas fêmeas que já recebiam a alimentação $a d$ libitum houve apenas a substituição da dieta.

Desse modo, conclui-se que o emprego da estratégia nutricional ad libitum possibilita a antecipação da inseminação para o segundo estro, sem redução no número de fetos, diminuindo-se os dias não produtivos da marrã. Porém, essa estratégia reduz a taxa de sobrevivência fetal até o $35^{\circ}$ dia de gestação. 


\section{Referências}

ALMEIDA, F. R.; MAO, J.; NOVAK, S.; COSGROVE, J. R.; FOXCROFT, G. R. Effects of different patterns of feed restriction and insulin treatment during the luteal phase on reproductive, metabolic and endocrine parameters in ciclic gilts. Journal of Animal Science, v. 79, n. 1, p. 200-212, 2012.

BELTRANENA, E.; AHERNE, F. X.; FOXCROFT, G. R.; KIRKWOOD, R. N. Effects of pre- and pos pubertal feeding on production traits at first and second estrus in gilts. Journal of Animal Science, v. 69, n. 3, p. 886-893, 1991a.

BELTRANENA, E.; FOXCROFT, G. R.; AHERNE, F. X.; KIRKWOOD, R. N. Endocrinology of nutritional flushing in gilts. Canadian Journal of Animal Science, v. 71, n. 4, p. 1063-1071, $1991 \mathrm{~b}$.

BOOTH, P. J.; COSGROVE, J. R.; FOXCROFT, G. R. Endocrine and metabolic responses to realimentation in feed-restricted prepubertal gilts association among gonadotropins, metabolic hormones, glucose, and uteroovarian development. Journal of Animal Science, v. 74, p. 840-848, 1996.

BURNETT, P. J.; WALKER, N. The effect of age and growth traits on puberty and reproductive performance in the gilt. Animal Production, v. 46, n. 3, p. 427-436, 1988.

CHAMBERLAIN, T. S.; HUGHES, P. E. The influence of mating frequency and nutrition on the stimulus value of boars. Animal Reproduction Science, v. 43, n. 2, p. 151-160, 1996.

CHRISTENSON, R. K. Ovulation rate and embryonic survival in Chinese Meishan and white crossbred pigs. Journal of Animal Science, v. 71, n. 11, p. 3060-3066, 1993.

COSGROVE, J. R.; CHARLTON, S. T.; COSGROVE, S. J.; ZAK, W.; FOXCROFT, G. R. Interactions between nutrition and reproduction in the pig. Reproduction in Domestic Animals, v. 30, n. 4, p. 193-200, 1995.

EDWARDS, R. L.; OMTVEDT,E. T.;TUESMAN, E. J.;STEPHENS, D. F.; MAHONEY, G. W. A. Reproductive performance of gilts following heat stress prior to breeding and in early gestation. Journal of Animal Science, v. 27, n. 6, p. 1634-1637, 1968.

FAILLACE, L. S.; BIGGS, C.; HUNTER, M. G. Factors affecting the age at onset of puberty, ovulation rate and time of ovulation in Chinese Meishan gilts. Journal of Reproduction and Fertility, v. 100, n. 2, p. 353-357, 1994.

FOXCROFT, G. R. Mechanism mediating nutritional effects on embryonic survival in pigs. Journal of Reproduction and Fertility, v. 52, p. 47-61, 1997.

FOXCROFT, G.; AHERNE, F. Management of the gilt and first parity sow: Part II. Selecting gilts for improved lifetime performance. In: SIMPÓSIO INTERNACIONAL DE REPRODUÇÃO E INSEMINAÇÃO ARTIFICIAL EM SUÍNOS, 7., 2000, Foz do Iguaçu. Anais... Foz do Iguaçu: Animalworld, 2000. p. 91-98.

HUGHES, P. E. Factors affecting the natural attainment of puberty in the gilt. In: COLE, D. J. A.; FOXCROFT, G. R. Control of pig reproduction. London: Butterwoths, 1982. p. 117-138.
KEMP, B.; SOEDE, N. M. Reproductive problems in primiparous sows. In: INTERNATIONAL PIG VETERINARY SOCIETY, 18., 2004, Hamburgo. Proceedings... Hamburg: Pig Veterinary Society, 2004. v. 2, p. 843-848.

KIM, S. W.; EASTER, R. A. Nutrient mobilization from body tissues as influenced by litter size in lactating sows. Journal of Animal Science, v. 79, n. 8, p. 2179-2186, 2001.

LAGO, V.; VIANNA, W. L.; GAMA, R. D.; ROSSETO, A. C.; PINESE, M. E.; MORETTI, A. M. Sincronização hormonal de estro e flushing alimentar em marrãs pós-púbere. In: CONGRESSO LATINO AMERICANO DE SUINOCULTURA, 2., 2004, Foz do Iguaçu. Anais... Foz do Iguaçu: Animalworld, 2004. p. 312.

LE COZLER, Y.; RINGMAR-CEDERBERG, E.; JOHANSEN, S.; DOURMAD, J. Y.; STERN, S. Effect of feeding level during rearing and mating strategy on performance of Swedish Yorkshire sows. Part 1. Growth, puberty and performances at service. Animal Science, v. 68, n. 3, p. 355-363, 1999.

NEWTON, E. A.; MAHAN, D. C. Effect of feed intake during late development on pubertal onset and resulting body composition in crossbred gilts. Journal of Animal Science, v. 70, n. 12, p. 37743780, 1992.

RHODES, M. T.; DAVIS, D. L.; STEVENSON, J. S. Flushing and altrenogest affect litter. Journal of Animal Science, v. 69, n. 1, p. 34-40, 1991.

SAS INSTITUTE. SAS User's guide: Statistical Analysis System, release 6.12. Cary: SAS Institute, 1998.

TANTASUPARUK, W.; LUNDEHEIM, N.; DALIN, A. M.; KUNAVONGKRIT, A.; EINARSSON, S. Weaning-to-service interval in primiparous sows and its relationship with longevity and piglet production. Livestock Production Science, v. 69, n. 2, p. 155-162, 2001.

THAKER, M. Y. C.; BILKEI, G. Lactation weight loss influences subsequent reproductive performance of sows. Animal Reproduction Science, v. 88, n. 304, p. 309-318, 2005.

VAN WETTERE, W. H.; REVELL, D. K.; MITCHELL, M.; HUGHES, P. E. Increasing the age of gilts at first boar contact improves the timing and synchrony of the pubertal response but does not affect potential litter size. Animal Reproduction Science, v. 95, n. 102, p. 97-106, 2006.

VIANNA, W. L.; PINESE, M. E.; CAMPOS ROSSETO, A. de; BOMBONATO, P. P.; RODRIGUES, P. H. M.; MORETTI, A. S. Relationship between prenatal survival rate at 70 days of gestation and morphometric pa of vagina, uterus and placenta in gilts. Reproduction in Domestic Animals, v. 39, n. 6, p. 381-384, 2004.

YOUNG, L. D. Reproduction of $3 / 4$ white composite and $1 / 4$ Duroc, $1 / 4$ Meishan, $1 / 4$ Fengjing or $1 / 4$ Minzhu gilts and sows. Journal of Animal Science, v. 76, n. 6, p. 1559-1567, 1998. 